\title{
O ENFERMEIRO NO CUIDADO À FAMÍLIA DO PACIENTE ONCOLÓGICO: UMA REVISÃO BIBLIOGRÁFICA
}

The nurse in care for the cancer patient's family: a bibliographic review

Wanderson Alves Ribeiro ${ }^{1}$

Marilda Andrade ${ }^{2}$

Bruna Porath Azevedo Fassarella ${ }^{3}$

Luiz Cláudio da Cruz Faria ${ }^{4}$

Mesulemete do Nascimento Martins ${ }^{5}$

Monique Lopes Gomes 6

João Luiz Ramos de Souza ${ }^{7}$

Juliana Mendes da Silva ${ }^{8}$

1. Enfermeiro. Mestrando Acadêmico em Ciências do Cuidado em Saúde pela Escola de Enfermagem Aurora de Afonso Costa da UFF, Niterói/RJ. Docente do Curso de Graduação da UNIG. Preceptor Acadêmico do Curso de Graduação em Enfermagem da UNIABEU. E-mail: nursing war@hotmail.com.

2. Enfermeira. Doutora em Enfermagem. Vice-Diretora, Professora Associada Escola de Enfermagem Aurora Afonso Costa da UFF, Niterói/RJ. E-mail: marildaandrade@uol.com.br.

3. Enfermeira. Mestranda em Ciências Aplicadas em Saúde da Universidade Severino Sombra. E-mail: brunaporath@gmail.com.

4. Enfermeiro. Graduado pela Universidade Iguaçu. E-mail: mesumoniqueluiz@gmail.com

5. Enfermeira. Graduado pela Universidade Iguaçu. E-mail: mesulemetemb@gmail.com

6. Enfermeira. Graduado pela Universidade Iguaçu. E-mail: moniquelopesgomes@hotmail.com

7. Enfermeiro. Pós-Graduado Em Processos Educacionais na Saúde com ênfase em metodologia ativa pelo IEP Sírio Libanês. E-mail: joaoluiz_100@yahoo.com.br

8. Enfermeira. Residente em Saúde da Família pela UFRJ. E-mail: julianameenders@gmail.com 


\section{Resumo}

O câncer se caracteriza por uma proliferação anormal de determinados tecidos, a qual escapa ao controle do organismo, tendendo à autonomia e geralmente causando efeitos extremamente agressivos sobre o corpo. Tem-se como objetivo descrever a relevância do enfermeiro no cuidado ao familiar do paciente oncológico. Trata-se de uma revisão bibliográfica de abordagem qualitativa. Para tal utilizou-se a Biblioteca Virtual de Saúde (BVS), nas seguintes bases de informações: LILACS; MEDLINE e SCIELO no período de janeiro a junho de 2018. Nesse sentido, a postura que o enfermeiro vai adotar precisa começar já no momento do diagnóstico, no sentido de aliviar o impacto de uma notícia deste tipo, esclarecendo sobre a ampliação das possibilidades de sobrevida e os ganhos possíveis em termos de qualidade de vida nos dias atuais, garantindo ao paciente que ele não estará solitário no enfrentamento do problema e de suas consequências, agindo também no sentido de estimular a esperança como condição fundamental para um tratamento exitoso, entre outras intervenções eficientes e humanizadas, como a dedicação total ao tratamento, a manutenção da autoestima, os benefícios de planejar um futuro ativo e positivo. Conclui-se o enfermeiro necessita identificar e avaliar suas próprias concepções relativas à doença e à interrupção da existência física, para que possa manter seu equilíbrio e estabelecer estratégias de enfrentamento, através da elaboração de metas claras e efetivamente significativas a serem direcionadas ao paciente e à família.

Descritores: Câncer; Enfermagem; Família.

\section{Abstract}

Cancer is characterized by an abnormal proliferation of certain tissues, which is beyond the control of the organism, tending to autonomy and generally causing extremely aggressive effects on the body. The purpose of this study is to describe the relevance of the nurse in the care of the family member of the cancer patient. This is a bibliographical review of a qualitative approach. For this purpose, the Virtual Health Library (VHL) was used in the following information bases: LILACS; MEDLINE 
and SCIELO in the period from January to June 2018. In this sense, the posture that the nurse is going to adopt must begin at the moment of the diagnosis, in order to alleviate the impact of such news, clarifying about the expansion of the possibilities of survival and the possible gains in terms of quality of life in the present day, assuring the patient that he will not be alone in facing the problem and its consequences, also acting to stimulate hope as a fundamental condition for a successful treatment, among other interventions efficient and humane, such as total dedication to treatment, maintaining self-esteem, the benefits of planning an active and positive future. It is concluded that nurses need to identify and evaluate their own conceptions regarding the disease and the interruption of physical existence, so that it can maintain its equilibrium and establish coping strategies, through the elaboration of clear and effectively significant goals to be directed to the patient and the family.

Keywords: Cancer; Nursing; Family.

\section{Introdução}

O câncer se caracteriza por uma proliferação anormal de determinados tecidos, a qual escapa ao controle do organismo, tendendo à autonomia e geralmente causando efeitos extremamente agressivos sobre o corpo. ${ }^{1}$

A manutenção de organismos multicelulares depende do equilíbrio entre suas células. Quando estas estão sendo produzidas em excesso, elas começam a morrer, num processo passivo de caráter degenerativo onde elas perdem suas funções metabólicas. A morte das células pode se dar por: apoptose, autofagia, necrose, mitose catastrófica e por senescência. Alterações na coordenação desses tipos de morte celular estão envolvidas na oncogênese. A apoptose, especificamente, é um programa de morte celular regulado e eficiente, sendo considerado um mecanismo natural de defesa antineoplásica. Inclusive, esse tipo de morte celular vem sendo considerado no tratamento das neoplasias malignas, eliminando células supérfluas ou defeituosas. ${ }^{2}$

O câncer, também chamado de neoplasia, pode ser de tipos diferenciados. Há o tipo benigno, que recebe o nome da célula de origem acrescida do sufixo OMA (Ex.: osteoma). Pode ser maligno. Quando atinge o tecido mesenquimal são 
denominados Sarcoma (osteosarcoma); Quando atinge o tecido epitelial são denominados Carcinomas. Caso atinja o tecido epitelial convencional de revestimento, utiliza-se o termo carcinoma como prefixo seguido do nome do tecido epitelial de origem (carcinoma basocelular). Caso atinja o epitélio glandular, o tumor recebe o prefixo adenocarcinoma mais o tecido epitelial de origem (adenoma hepatocelular). Há ainda, o tipo de tumor potencialmente maligno, chamado também de "Tumor Borderline". Este último merece uma explicação um pouco mais detalhada, por apresentar características de benignidade, mas, com tendência à malignidade (Leiomiomas). ${ }^{2}$

Diversas classes de neoplasias apresentam grupos de tumores com características intermediárias entre benignas e malignas, comportamento que não pode ser claramente previsto pelo patologista ou oncologista, sendo a contagem de mitoses o principal critério para definir o comportamento da neoplasia e as opções de tratamento para estes casos. ${ }^{3}$

Independente da fase em que o câncer é descoberto torna-se indispensável saber qual a extensão dos danos causados ao organismo. O método utilizado para a classificação é chamado de estadiamento e sua importância reside na constatação do grau de propagação da doença, o que caracteriza seu estágio evolutivo no organismo. O estadiamento pode ser tanto clínico como patológico. ${ }^{1}$

De acordo com o INCA (Instituto Nacional do Câncer), o tipo clínico é estabelecido a partir dos dados do exame físico e dos exames complementares pertinentes ao caso. O tipo patológico baseia-se nos achados cirúrgicos e no exame anatomopatológico da peça operatória. A classificação em grupos obedece a certas variáveis, tais como: localização, tamanho ou volume do tumor, invasão direta e linfática, metástases à distância, diagnóstico histopatológico, produção de substâncias, manifestações sistêmicas, duração dos sinais e sintomas, sexo e idade do paciente, entre outras. Para isto, existem regras internacionalmente estabelecidas e que se encontram em constante atualização. ${ }^{1}$

O câncer pode surgir em qualquer parte do organismo, sendo que cada órgão pode ser acometido por diferentes tipos de tumor. Uns mais agressivos outros menos. Tendo em vista que um órgão pode apresentar vários tipos histológicos de tumor, é apropriado que os sistemas de estadiamento variem de acordo com a 
classificação histopatológica do mesmo. Não raro, o estadiamento só pode ser estabelecido através de procedimentos cirúrgico-terapêuticos, como, por exemplo, nos casos de tumor de ovário, no qual é indicada cirurgia para ressecção do tumor e inventário das condições da cavidade abdominal. Donde se deduz que, a indicação do tratamento vai depender do estadiamento da doença, pois um estadiamento bem conduzido favorece a opção por condutas terapêuticas mais eficazes e adequadas para cada caso. ${ }^{1}$

Os vários tipos de câncer são classificados de acordo com a localização primária do tumor. Exemplos: câncer do colo do útero, da cavidade oral, do esôfago, de cólon e reto, de laringe, traqueia, câncer de mama, câncer de próstata, bexiga, sistema nervoso central, câncer da tireoide, de pulmão, linfoma de Hodgkin, linfoma de não Hodgkin, leucemias e outros. ${ }^{4}$

Salienta-se que o diagnóstico do câncer por si só tem um efeito devastador não só na vida do paciente, mas também na de sua família, gerando os sentimentos mais controversos, como por exemplo: o medo do desconhecido, a incerteza acerca da capacidade profissional da equipe médica, da adequação quanto à estrutura tecnológica da instituição de saúde para prestar o atendimento, do próprio tratamento de escolha e a sua genuína eficácia. ${ }^{5}$

Alie-se a isso, ainda, o temor das mutilações e desfigurações, a expectativa da terminalidade da vida, a incerteza das perdas decorrentes de todo o contexto patológico, seja nas esferas emocional, social, profissional, financeira, afetiva, entre outras que quase sempre ocorrem. Por isso, é importante falar da doença em si, sem reservas e de forma técnica e muito esclarecedora para, aí sim, adentrar a esfera do humano e ajudar a família no enfrentamento destas questões. ${ }^{5}$

O sofrimento da pessoa que recebe esse diagnóstico envolve de forma extremamente impactante os seus familiares. Aliás, o câncer é uma doença familiar por definição, não somente sob o ponto de vista genético, mas, sobretudo pela perturbação que provoca na estrutura cotidiana de sua parentela. Quando um membro da família é diagnosticado, todos os familiares são invariavelmente afetados, em função do temor da morbi-mortalidade e do estigma causado pela doença. Portanto, o cuidado com a família se torna indispensável, pois as 
consequências biopsicossociais que esta patologia promove na engrenagem familiar são deveras traumáticas e muito difíceis de enfrentar sem um apoio adequado. ${ }^{6}$

Quando se abordava contexto de saúde costumava-se decair em um modelo assistencial onde o alvo era trucidar as doenças e suas sintomatologias e com isso, o portador da patologia era visto apenas como um diagnóstico, dificultando o processo de cuidar e oferecer informações com a proposta de subsidiar o indivíduo em sua própria recuperação e tratamento. ${ }^{7}$

Portanto, o profissional enfermeiro é habilitado e capacitado para prestar cuidado ao cliente e sua família em todas suas esferas existenciais, considerando as necessidades curativas, preventivas e educativas de cuidados em saúde. Assim sendo, o objeto deste estudo é o enfermeiro no suporte humanizado à família do paciente oncológico dentro do contexto do enfrentamento desta morbidade. ${ }^{8}$

Para tal, traçou-se a seguinte questão norteadora: Qual a relevância do enfermeiro no cuidado ao familiar do paciente oncológico?

Nesse sentido, a pesquisa tem como objetivo: descrever a relevância do enfermeiro no cuidado ao familiar do paciente oncológico.

\section{Metodologia}

Trata-se de uma pesquisa bibliográfica de abordagem qualitativa e caráter descritivo. Cabe ressaltar que a pesquisa bibliográfica que é desenvolvida com auxílio de material já elaborado, constituído principalmente de livros e artigos científicos. Contudo em grande parte dos estudos seja exigido algum tipo de trabalho deste gênero, há pesquisas desenvolvidas exclusivamente a partir de fontes bibliográficas. $^{9}$

Com vista a conhecer a recorrência e o estado da temática, foi realizada uma pesquisa bibliográfica para embasamento e contextualização do tema em questão.

Os dados foram coletados em base de dados virtuais. Para tal utilizou-se a Biblioteca Virtual de Saúde (BVS), nas seguintes bases de informações: Literatura Latino-Americana e do Caribe em Ciências da Saúde (LILACS); Medical Literature Analysis and Retrieval System Online (MEDLINE) e Scientific Electronic Library 
Online (SCIELO) no período de janeiro a junho de 2018, além de material impresso como livros e artigos de revistas periódicas da saúde.

Optou-se pelos seguintes descritores: Câncer; Enfermagem; Família que se encontram nos Descritores em Ciência da Saúde (DECS). Estabeleceu-se então para a realização da pesquisa os critérios de inclusão: textos na íntegra e em português com abordagem da temática estabelecida e que obedecessem ao recorte temporal de 2007 a 2017 e como critérios de exclusão, os textos incompletos e em língua estrangeira, textos que não abordassem a temática estabelecida e com recorte temporal inferior a 2007, com seleção de 13 artigos.

Cabe mencionar que os textos em língua estrangeira foram excluídos devido o interesse em embasar o estudo com dados do panorama brasileiro e os textos incompletos, para oferecer melhor compreensão através da leitura de textos na integra.

\section{Analise de Dados e Discussão de Resultados}

Posterior à leitura reflexiva dos ensaios supracitados emergiram três categorias: A Família como Suporte do Cuidado; O Paciente Oncológico na Ótica da Enfermagem; O Enfermeiro no Contexto do Cuidado ao Familiar.

\section{1a Categoria: A Família como Suporte do Cuidado}

Cada família possui nos meandros de sua convivência representações, significações e simbolismos presentes em seu histórico familiar, o que dá o tom às ações de seus membros, tanto dentro quanto fora do grupo familiar. Porém, é oportuno mencionar que o apoio da família se faz prioritário, quando um dos seus encontra-se com uma doença grave, como o câncer. Os familiares tornam-se singulares atores de uma história nada agradável e que requer a superação de questões anacrônicas para serem consideradas e muito menos expurgadas no momento, que exige de cada um alto grau de compreensão, generosidade e doação. ${ }^{10}$ 
A família está, juntamente com seu ente querido, vivenciando e tendo que lidar com uma situação cheia de incertezas e com a temível expectativa da terminalidade da vida. É natural que todos sofram com esses sentimentos, mas é de vital importância verbalizá-los, transformá-los em linguagem para que não se transformem em doença, no futuro. Conversar sobre o assunto ajuda a amenizar o estresse produzido pelo contexto penoso que estão experimentando. ${ }^{11}$

Os processos emocionais desencadeados nesses pacientes exigem um profissional especializado É fundamental compreender e dar suporte a essas transformações inesperadas, bem como ouvir e aprender com o paciente, tendo sempre em mente que se está cuidando de um ser humano e não apenas da enfermidade que ele traz. Diante de uma patologia como o câncer, sabe-se que o envolvimento familiar é inevitável. ${ }^{12}$

Cada uma das imagens concebidas sobre família figura valores, define funções, e, obviamente, papéis sociais. Embutidas nestas imagens estão a "realidade" própria de cada "família", além de outros aspectos como seus conflitos internos, suas preferências por um ou outro membro, confidencialidades, dilemas. Tais realidades devem ser compreendidas em suas singularidades e não tendo em vista uma "realidade genérica da família perfeita", ainda que diante da doença. Há que se considerar ainda a diversidade de tipos de "famílias" nas sociedades contemporâneas. ${ }^{13}$

A família é o primeiro e o mais fundamental grupo social, dentro do qual o ser humano começa a desenvolver suas primeiras relações afetivas, cria vínculos e internaliza princípios e valores para a formação do caráter no futuro. Essa relação familiar apresenta-se de forma vinculada, que é como se um fosse a extensão do outro, pois acredita-se que a experiência de uma doença grave traz modificações no modo de pensar, sentir e agir das pessoas. Por isso, é que o câncer é considerado uma doença familiar por essência. ${ }^{14}$

\section{2a Categoria: O Paciente Oncológico na Ótica da Enfermagem}

Quando se trata de um paciente terminal, os complicadores multiplicam-se, pois todos se confrontam com seus próprios limites, seus temores, as experiências 
pregressas, vulnerabilidades, se veem refletindo sobre o significado da própria vida que estão vivendo, o que pode resultar em negação e fuga da realidade. ${ }^{15}$

Este tipo de paciente, necessitado de cuidados paliativos exige da equipe de enfermagem uma abordagem diferenciada, cujo enfoque encontra-se no alívio do sofrimento, na satisfação das necessidades básicas, no controle dos sintomas, sem uma função nem diagnóstica nem curativa, com vistas apenas à preservação da qualidade de vida e da dignidade da pessoa humana até o fim de sua existência, complementam. ${ }^{15}$

O enfermeiro é o profissional que está sempre presente ao lado do paciente e também aquele que está mais próximo da família, prestando aos primeiros, os cuidados diretos e a estes últimos o apoio emocional de que tanto necessitam, o que provavelmente faz desencadear um envolvimento afetivo entre os atores envolvidos. Este é concebido como sendo uma forma de comportamento em que uma pessoa mantém a proximidade com outra, que é diferente e preferida. É considerado como uma base de segurança e quando por algum motivo é interrompido, como o que acontece com a morte, gera sofrimento e provoca o luto, que é uma resposta natural e esperada. ${ }^{16}$

A literatura afirma que o estado terminal é a condição em na qual já não lhe é mais possível curar o paciente, mas apenas e tão somente cuidar. Os cuidados ao paciente terminal representam um grande desafio para os enfermeiros que devem reconhecer que, quando as metas do curar deixam de existir, as metas do cuidar devem ser reforçadas. ${ }^{16}$

O enfermeiro em face de sua impotência diante do inexorável deve estar devidamente preparado, para adotar algumas medidas diferenciadas a fim de ajudar a pessoa a morrer com dignidade. $O$ agir da equipe de enfermagem em relação ao paciente e à família assume dimensões muito mais complexas, suscitando conceitos e atitudes que requerem um trato muito mais humanizado, envolvendo várias esferas de saber mediante a perspectiva da terminalidade da vida, principalmente em culturas como a ocidental, onde a morte permanece um tabu, um tema cuja menção deve ser evitada a todo custo, lecionam. ${ }^{11}$

Existe até mesmo uma resistência dos médicos em diagnosticar um paciente como terminal dado o fato de se tratar de um diagnóstico demasiadamente 
categórico, definitivo, que exige alta capacidade técnico-científica, precisão e segurança, haja vista que se está assumindo uma grande responsabilidade que envolve a vida e os sentimentos de muitas pessoas já bastante fragilizadas. Existe ainda a remota possibilidade do diagnóstico não se confirmar com a evolução do caso, colocando os médicos em uma situação constrangedora, em que a eventual melhora do paciente, esboça a feição da incompetência do profissional em diagnosticar. ${ }^{17}$

Com efeito, diagnosticar a inexistência de perspectivas de cura terapêutica, é atribuição deveras angustiante para todos. Desta forma, a decisão de não mais investir no paciente, no sentido terapêutico, visando a cura, nunca é tomada por um profissional isoladamente: sempre é feita por uma equipe multidisciplinar, onde a família deve ser sempre ouvida com muito respeito e receber assistência psicológica para enfrentar a situação, conforme corroboram as supracitadas autoras. ${ }^{17}$

\section{3a Categoria: O Enfermeiro no Contexto do Cuidado ao Familiar}

No cerne do problema abordado neste projeto encontra-se a importância da atuação da equipe de enfermagem no apoio à família do paciente tanto nos momentos de impacto frente ao diagnóstico, como durante o tratamento, mas também diante da realidade, algumas vezes remanescente, dos cuidados paliativos, ou seja, aqueles indicados aos casos sem possibilidade de cura, assim como do óbito do paciente. Estes casos teoricamente sugerem a capacidade do enfermeiro em executar um trabalho extremamente técnico e isento de envolvimento emocional, contudo, o enfermeiro também é um ser humano susceptível aos mesmos agravos em relação à sua saúde tanto quanto à de sua família. ${ }^{11}$

A família e seu ente querido sob cuidados paliativos, ao longo do tempo, constroem com a equipe, mormente com a enfermagem por estar sempre muito mais próxima, uma conexão genuína baseada na confiança, no apoio e na compreensão, tornando este distanciamento, na prática, bem mais complicado do que a teoria fleumática faz parecer. ${ }^{11}$

Muitas vezes, o familiar projeta os pensamentos além do momento presente, e visualiza que as possibilidades que o futuro the reserva podem ser sofridas, pois 
os planos para o futuro ficam ameaçados de não acontecer. Isso porque o câncer é uma doença percebida no senso comum como incurável, perigosa e horrível, além de provocar a morte após longo sofrimento e com isso transtornos em todo o contexto familiar. ${ }^{17}$

Muitos tipos de câncer, atualmente, já não representam uma sentença de morte. Além disso, ainda há a questão da precocidade com a qual o tumor é detectado e que é iniciado o tratamento. Os avanços terapêuticos são bastante significativos, elevando a taxa de sobrevida e também de qualidade de vida. Porém, o estigma de doença devastadora, mutiladora e fatal permanece. ${ }^{18}$

Nesse sentido, a postura que o enfermeiro vai adotar precisa começar já no momento do diagnóstico, no sentido de aliviar o impacto de uma notícia deste tipo, esclarecendo sobre a ampliação das possibilidades de sobrevida e os ganhos possíveis em termos de qualidade de vida nos dias atuais, garantindo ao paciente que ele não estará solitário no enfrentamento do problema e de suas consequências, agindo também no sentido de estimular a esperança como condição fundamental para um tratamento exitoso, entre outras intervenções eficientes e humanizadas, como a dedicação total ao tratamento, a manutenção da autoestima, os benefícios de planejar um futuro ativo e positivo. ${ }^{18}$

$\mathrm{Na}$ era do conhecimento torna-se muito importante a busca de novas competências nas formas de organização do trabalho em saúde, nas atitudes profissionais integradas aos sistemas sociais de relações e interações múltiplas, em suas diversas dimensões, abrangências e especificidades. Inovando formas, práticas e métodos de produção de conhecimento em saúde é necessário levar em consideração os diferentes olhares, vivências profissionais e também que se estabeleçam novas e sempre mais significativas relações e interações profissionais para entender e, por conseguinte, tratar o ser humano de forma mais ampla e integral. ${ }^{19}$

O assistir em oncologia é um trabalho de extrema importância e abrangência para o enfermeiro, indo muito além de um esforço por estender a expectativa de vida do paciente. Ainda que perdure o modelo biomédico e hospitalocêntrico, a essência mais visceral do assistir um ser humano em uma situação de extrema vulnerabilidade, como é o caso do paciente oncológico, supõe uma relação 
biopsicossocial e cultural e por que não dizer filosófica, na medida em que envolve além dos conhecimentos técnicos e científicos, a questão do agir respeitando o ser em sua totalidade, em sua complexidade, procurando resguardar a autonomia do paciente, manter o seu desejo de viver, mas de viver com qualidade, de descobrir a partir da própria experiência com o câncer, dons, virtudes, sonhos até então desconhecidos dele próprio. Esse olhar positivo, original, curioso sobre a própria existência supõe uma relação de empatia entre aquele que cuida e aquele que é cuidado. ${ }^{20}$

Os significados construídos entre a enfermagem o paciente e a família do mesmo evidenciam a necessidade de ampliar o foco de atenção dos profissionais de saúde, de modo a incluir as referências socioculturais no contexto da vida comum das pessoas que, naquele momento estão vivenciando a experiência dramática de ter que enfrentar uma neoplasia maligna e todos os seus desdobramentos. ${ }^{21}$

Encarar a luta contra o câncer como uma trajetória de autodescoberta, de autoconhecimento pode, ante um olhar mais atento, ser um processo de mútuo cuidar, de mútuo aprender, de mútua transformação, onde a família precisa entender a abordagem e se dispor a um grande envolvimento, onde a palavra de ordem não é morte. É vida. Seu ente querido está vivo e a família é parte essencial e cobeneficiária de toda a pluralidade de vivências deste processo. ${ }^{21}$

O estímulo ao desenvolvimento deste olhar sobre a vida, ainda que com a presença da morbidade e todas as circunstâncias que a envolvem é fundamental porque mesmo após o término do tratamento, ainda que exitoso, o paciente continua a conviver com o medo da recidiva e com as sequelas que sobrevêm de um tratamento oncológico, que é mais que um processo terapêutico complexo. É uma experiência pessoal onde a melhoria da qualidade de vida passa a ser um dos resultados esperados tanto das práticas assistenciais quanto do próprio fim do tratamento. Para a enfermagem, quanto ao cuidado percebido como um sentimento de realização ou gratificação, o mesmo evidencia-se no momento em que esse paciente sai curado ou consegue restabelecer-se com sucesso. ${ }^{20}$

O câncer altera as relações entre os membros da família, traz à tona sentimentos esquecidos ou pouco demonstrados ao mesmo tempo em que altera conceitos e visões que as pessoas têm a respeito de seus familiares em situações 
normais. Esse quadro vai mudando à medida que as pessoas da família vão adquirindo conhecimento sobre a doença, sobre a forma de lidar com o ente querido adoecido. $^{22}$

Os familiares aprendem também a reorganizar sua própria vida com o fim de oferecer suporte à vida cotidiana do paciente, porque mesmo que este esteja temporariamente capacitado a desempenhar seus papéis sociais, a rotina segue em frente. A vida não vai parar porque a pessoa adoeceu. É aí que a família começa a se organizar e flexibilizar seu próprio tempo, no sentido de criar estratégias capazes de superar as adversidades, dedicando tempo e energia para auxiliá-la na rotina. Neste resgate biopsicossocial dos envolvidos numa doença complexa e sofrida como a neoplasia maligna, nesta releitura do grupo enquanto família está a participação do enfermeiro, quando ele transpõe o cuidar meramente biologicista e adentra a esfera do humano apoiando o outro que sofre. ${ }^{22}$

Compreender a complexidade que é ter um enfermo grave na família para lidar, é fundamental para que se possa direcionar a intervenção da equipe de saúde, objetivando respostas que contemplem as demandas singularizadas de cada paciente e de cada família, porque a experiência da doença é única para cada ser humano, e reconhecendo que elas expressam necessidades coletivas que são expressões de determinantes sociais, econômicos e culturais que impõem condições de vida adversas para determinados segmentos da população. ${ }^{22}$

\section{Conclusão}

Conclui-se o enfermeiro necessita identificar e avaliar suas próprias concepções relativas à doença e à interrupção da existência física, para que possa manter seu equilíbrio e estabelecer estratégias de enfrentamento, através da elaboração de metas claras e efetivamente significativas a serem direcionadas ao paciente e à família. Estratégias que contemplem tanto os aspectos biopsicossociais como também o aspecto espiritual de forma equânime e absolutamente respeitosa, visando uma assistência adequada que possibilite minimizar o sofrimento de todos os envolvidos no processo, inclusive o do próprio profissional. 
Nesse sentido, pacientes e familiares devem ser reconhecidos, pelo enfermeiro, como atores sociais ativos, participantes, corresponsáveis em todo o processo, desde o impactante diagnóstico, durante o tratamento que é, sem dúvida muito difícil para todos até o desfecho, seja qual for, respeitando-se as peculiaridades emocionais, socioeconômicas, religiosas e culturais que impõem limites para o enfrentamento à situação do câncer.

Por fim, tal nível de atuação exige uma agregação de saberes, tendo como diretriz a perspectiva de atenção integral e humanizada, que envolva pacientes e familiares, possibilitando a construção de um trabalho coeso, interdisciplinar, apontando questões relevantes que venham a produzir conhecimentos voltados para as demandas apresentadas pelos usuários e famílias tendo sempre como objetivo precípuo a excelência na qualidade da assistência ao paciente oncológico e sua família.

\section{Referências}

1. BRASIL, Ministério da Saúde. Instituto Nacional do Câncer (INCA). Ações de enfermagem para o controle do câncer: uma proposta de integração ensinoserviço. $3^{\mathrm{a}}$ ed. Rio de Janeiro, 2012. Disponível em:<http://www.inca.gov.br>. Acesso em> Ago. 2017.

2. GRIVICICH, Ivana et al. Morte celular por apoptose. Revista brasileira de cancerologia, v. 53, n. 3 , p. 335-343, 2007. Disponível em: < http://pepsic.bvsalud.org/scielo>. Acesso em Ago. 2017.

3. NICIDA, Lucia Regina de Azevedo. História da política de prevenção e controle do câncer no Amazonas, 1974 - 2011. 2015. 147 f. Dissertação (Mestrado em Saúde, Sociedade e Endemias na Amazônia) - Universidade Federal do Amazonas, Manaus, 2015. 
4. FACINA, Taís. Estimativa 2014-Incidência de Câncer no Brasil. Rev Bras Cancerol, v. 60, n. 1, 2014. Disponível em <http://www.scielo.br>. Acesso em: Ago. 2017.

5. SILVA, Rita de Cássia Velozo da; CRUZ, Enêde Andrade da. Planejamento da assistência de enfermagem ao paciente com câncer: reflexão teórica sobre as dimensões sociais. Esc. Anna Nery, Rio de Janeiro , v. 15, n. 1, p. 180185, Mar. 2011 . Disponível em: <http://www.scielo.br/scielo> Acesso em: Out. 2017.

6. BARRETO, Thamara Sena; AMORIM, R. da C. A família frente ao adoecer e ao tratamento de um familiar com câncer. Rev Enferm UERJ, v. 18, n. 3, p. 462-7, 2010.

7. Ribeiro, WA; Mariano, ES; Cirino, HP; Teixeira, JM; Martins, LM; Andrade, M. Educação em saúde aos portadores de hipertensão arterial e diabete mellitus na Estratégia Saúde da Família. Revista Pró- UniverSUS. 2017 Jun./Dez.; 08 (2):110-114.

8. Ribeiro WA, Martins LM, Couto CS, Cirino HP, Teixeira JM, de Almeida VLA. Práticas educativas do enfermeiro na prevenção da gravidez na adolescência: estratégias e perspectivas. Revista Pró-UniverSUS. 2017 Jul./Dez ; 08 (2): 5862.

9. Gil, Antonio Carlos. Como elaborar projetos de pesquisa. 5. ed. São Paulo: Atlas, 2008.

10. OLIVEIRA, Márcia Cristina Lucas de; FIRMES, Maria da Penha Rodrigues. Sentimentos dos profissionais de enfermagem em relação ao paciente oncológico. REME rev. min. enferm, v. 16, n. 1, p. 91-97, 2012. Disponível em:< http://redalyc.org/articulo.> Acesso em: Set. 2017. 
11. AVANCI, Barbara Soares et al. Cuidados paliativos à criança oncológica na situação do viver/morrer: a ótica do cuidar em enfermagem. Esc Anna Nery Rev Enferm, v. 13, n. 4, p. 708-16, 2009.

12. MARCONI, Marina de Andrade; LAKATOS, Eva Maria. Fundamentos de Metodologia Científica. 7. ed. São Paulo: Atlas, 2010.

13. MARCON, Sonia Silva et al. Estratégias de cuidado a famílias que convivem com a doença crônica em um de seus membros - DOI: 10.4025/cienc cuid saude. V 8i0. 9720. Ciência, Cuidado e Saúde, v. 8, p. 70-78, 2010.

14. SOUZA, Maria das Graças Gazel de; ESPÍRITO SANTO, Fátima Helena do. O Olhar que Olha o Outro... Um Estudo com Familiares de Pessoas em Quimioterapia Antineoplásica. Rev bras de Canc, v. 54, n. 1, p. 31-41, 2008.

15. STUMM, E.M.F.; MILADI, Eniva et al. Vivências de uma Equipe de Enfermagem no Cuidado a Pacientes com Câncer, Paraná, COGITARE, 2008, n. 13, Jan/Mar. Disponível em:< http://redalyc.org/articulo.> Acesso em: Ago. 2017.

16. MENDES, Juliana Alcaires; LUSTOSA, Maria Alice; ANDRADE, Maria Clara Mello. Paciente terminal, família e equipe de saúde. Rev. SBPH, Rio de Janeiro, v. 12, n. 1, p. 151-173, jun. 2009. Disponível em: < http://pepsic.bvsalud.org/scielo>. Acesso em Set. 2017.

17. SANTOS, I. M. M., SANTOS, R. S. A etapa de análise no método história de vida - Uma experiência de pesquisadores de enfermagem. Revista Texto e Contexto Enfermagem. Florianópolis. v.17, n.4, 2008.

18. MENEZES, Maria de Fátima Batalha de et al. Câncer, pobreza e desenvolvimento humano: Desafios para a assistência de enfermagem em 
oncologia. Revista Latino Americano de Enfermagem. v. 28, 2007. Disponível em: < http://www.eerp.usp.br/rlae> Acesso em Jul 2017.

19. ZACCHÉ AVELLAR, Luziane; IGLESIAS, Alexandra; FERNANDES VALVERDE, Priscila. Sofrimento psíquico em trabalhadores de enfermagem de uma unidade de oncologia. Psicologia em estudo, v. 12, n. 3, 2007.

20. MANSANO-SCHLOSSER, Thalyta Cristina; CEOLIM, Maria Filomena. Qualidade de vida de pacientes com câncer no período de quimioterapia. Texto \& Contexto Enfermagem, v. 21, n. 3, 2012. Disponível em: <http://www.scielo.br/scielo> Acesso em: Set. 2017.

21. SILVA, M. E. D.C.; SILVA, L. D.C., DANTAS D. O. R.; DUARTE I. S.; SOUZA, J. F. M.: Assistência de enfermagem ao paciente oncológico no hospital: Revisão Integrativa. Rev Enferm UFPI. 2014; v. 2 (no esp.):69-7. Disponível em: < http://www.ojs.ufpi.br/index. php/reufpi/article/view/1359 > Acesso em Set. 2017.

22. FERREIRA, Noeli Marchioro Liston et al. Câncer e família: compreendendo os significados simbólicos-doi: 10.4025/cienc cuid saude. V 9i2. 8749. Ciência, Cuidado e Saúde, v. 9, n. 2, p. 269-277, 2010. 